\title{
Energy Efficient Traffic Offloading in Multi-tier Heterogeneous 5G Networks Using Intuitive Online Reinforcement Learning
}

\author{
Ismail AlQerm*, Member, IEEE and Basem Shihada**, Senior Member, IEEE
}

\begin{abstract}
The energy efficiency in multi-tier heterogeneous $5 G$ networks is a critical issue due to the fact that the macro base stations (BSs) power consumption is considerably high and proportional to their traffic load. Traffic offloading from macrocells to small cells is envisioned as a potential solution to improve energy efficiency in 5G heterogeneous networks. However, traffic offloading causes traffic congestion at small cells, interference as a result of small cells transmissions, and aggregate power consumption of small cells. These factors make the traffic offloading procedure more challenging. In this paper, we propose a conditional traffic offloading scheme, which relies on macrocells and small cells system load information to determine the most energy efficient traffic offloading strategy, select the proper operation mode of small cells, and fulfill macro users applications' quality of service $(\mathrm{QoS})$ requirements. The proposed scheme is developed using a novel intuitive online reinforcement learning methodology to perform the conditional traffic offloading in which each macro BS conjectures the offloading strategies of other macrocells. The convergence of the proposed scheme is proved and numerical results demonstrate its capability to achieve fast convergence with QoS guarantee and significant energy efficiency.
\end{abstract}

Index Terms-5G heterogeneous networks, Traffic offloading, Online reinforcement learning, Energy efficiency, macrocells, small cells

\section{INTRODUCTION}

Dense heterogeneous networks comprising macrocells and small cells contribute to the main trends in the future $5 \mathrm{G}$ to enhance the throughput of cellular networks at relatively low operational costs [1] [2]. The dense and random deployment of small cells in $5 \mathrm{G}$ heterogeneous networks raises fundamental challenges for the energy consumption of heterogeneous networks. With the perceived increase in energy consumption of about $40 \%$ for wireless cellular networks from 2010 to 2020 [3], lack of coordination of small cells and high operational costs of macrocells reflected by the energy consumed for the operations are crucial challenges that may limit the spread of the future heterogeneous $5 \mathrm{G}$ networks. Traffic offloading in multi-tier heterogeneous 5G networks, where small cells are exploited for handling the offloaded traffic from macrocells aims at allocating more capacity for services while maintaining QoS for the users [4] and boosting the energy efficiency.

*Ismail AlQerm is with Department of Mathematics and Computer Science at University of Missouri Saint Louis, St. Louis MO, United States. Email: alqermi@umsl.edu.

**Basem Shihada is with Division of Computer, Electrical, Mathematics Science and Engineering at King Abdullah University of Science and Technology, Thuwal, Saudi Arabia. Email: basem.shihada@kaust.edu.sa.
However, traffic offloading may cause traffic congestion at the small cells as they have distinct load. For example, some of the small cells may not be operational all the time and others may be overwhelmed with the offloaded traffic [5]. In addition, it increases the interference between the macrocells and other small cells. This leads to a severe increase in the energy consumption across the whole network. Despite the fact that small cells have a relatively lower power consumption profile, one of the major concerns in the future dense deployments is the high aggregated energy consumption.

To overcome these challenges, it is necessary for the macro base station (BSs) to be aware of each other's traffic offloading procedure to avoid interference, eliminate the traffic congestion possibility, and improve energy efficiency. Dense deployment of small cells should be carefully designed in order to avoid undesired network behavior. In addition, traffic in $5 \mathrm{G}$ heterogeneous networks is expected to vary spatially and temporally. This traffic variation will impact the performance of the 5G networks and the traffic processing techniques. Thus, it is necessary to find the optimal densities of small cells and determining which small BSs should be switched on/off according to the spatial and temporal traffic variation of users' traffic patterns. The relationship between the traffic and the small cells activity is more observable when the traffic becomes diversified and fluctuant [6] [7]. Hence, optimal traffic offloading and small BSs operation policies for dense $5 \mathrm{G}$ heterogeneous networks can play a key role in improving the energy efficiency and macro users data rate.

In this paper, we propose an energy efficient traffic offloading scheme that tackles the mentioned energy efficiency challenges and considers traffic variation. The proposed scheme offloads traffic from macrocells to small cells including picocells and femtocells under the condition that the cell system load of all the cells is maintained below certain threshold. The cell load is an essential factor that impacts energy efficiency as it grows with traffic demand and it contributes to inter cell interference and small cells traffic congestion. In addition, the cellular users' applications' QoS is also dependent on the cell load. For instance, when the cell load is low, BSs are able to offer their associated users a good capacity to meet their QoS requirements. To achieve the efficient traffic offloading and tackle the macro BSs offloading awareness issue, we develop an intuitive online reinforcement learning scheme, where each learning agent (macro BS) aims to perform traffic offloading by surmising other agents traffic offloading actions for certain network state without cooperation and information 
exchange. Hereby, a traffic offloading strategy is defined as a sequence of actions, and each action takes the form of small cells selection for offloading (e.g., switching on a small cell to offload the traffic demands within its respective coverage or offload traffic to an active small cell). Our task is to maximize the overall energy efficiency in the network while satisfying the constraint of flow-level QoS requirement. The reason for choosing online reinforcement learning to solve this optimization problem is that it is difficult to determine an exact state transition model for a system with large state space in such dynamic environment. Moreover, online reinforcement learning does not require any prior knowledge of the system's behavior. Thus, it is difficult to compute the optimal traffic offloading strategy through applying a model-based dynamic programming algorithms. The paper has the following contributions:

- The paper proposes traffic offloading scheme that exploits cell load as a driving factor to achieve energy efficient offloading as the cell load impacts inter-cell interference, small cell traffic congestion, and energy efficiency.

- Novel online reinforcement learning technique is developed to select an efficient traffic offloading strategy that maximizes the network energy efficiency.

- The proposed online reinforcement learning based scheme deals with the awareness of other macro BSs traffic offloading strategies by introducing the intuition feature to online learning with which the scheme has the ability to make each macro BS conjectures other macro BS offloading strategies to find a joint offloading action without explicit information exchange. This has a positive impact on the learning process as it reduces the scheme computation overhead and improves the selected action quality.

The rest of the paper is organized as follows. Related work is presented in section II. The system model and problem formulation are explained in section III. The intuitive online reinforcement learning approach including the learning model and the intuitive mechanism are described in section IV. Section $\mathrm{V}$ presents the numerical results that demonstrate the proposed approach capability to enhance energy efficiency while maintaining QoS of users. The paper concludes in Section VI.

\section{RELATED WORK}

Researchers' studies found that 25 to $30 \%$ of the total power can be saved by reducing the number of active macrocells when traffic is low [8] [9]. Recently, power savings in heterogeneous cellular networks through the usage of small cells to handle traffic offloaded from macrocells draw the attention of many researchers. A joint user association and small BS switching scheme was proposed in [10] to increase the system capacity and reduce power consumption. In [11], the authors investigated the small BSs dynamic operation to handle the offloaded traffic from the macro BSs in heterogeneous networks. They exploited joint location and user density based operation scheme to manage the dynamic operation of the small BSs. An optimal offloading scheme in a stochastic environment is proposed in [12]. The work in [13] proposed a joint optimization of traffic scheduling and power allocation that aims at minimizing the total on-grid power consumption of macro and small cells, while guaranteeing each served MU's traffic requirement. The authors in [14] proposed an energyaware traffic offloading scheme in which user associations, ON/OFF status of Small BSs, and power control are jointly optimized according to the statistical information of energy arrival and traffic load. The power saved by the activation of small BSs is derived in a closed form and the optimal amount of traffic to be offloaded is determined accordingly. D2D communication was exploited in [15] to assist the traffic offloading process in $5 \mathrm{G}$ heterogeneous network which aimed at improving the network capacity and mitigating the traffic congestion at the macro BS. The offloading problem was solved using dynamic programing as well as the theoretical performance upper bound of D2D assisted traffic offloading. [16] provided a study on the optimal Dual connectivity enabled traffic offloading through small cells that is powered by energy harvesting with the goal of minimizing the total ongrid power consumption of all small cells and macrocells. In [17], stochastic geometry was utilized to perform offloading of cyber physical systems to small cells which consequently can increase the capacity of the macrocells and release more network resources for users. The work in [18] proposed an optimization-based framework for energy efficient radio resource management in heterogeneous 5G networks. Specifically, the small BSs are activated with jointly optimized user association and spectrum allocation to stabilize the network. An optimal ON-OFF BS adaptation scheme was designed in [19] using stochastic geometry. The work in [20] proposed a multi-objective framework as an energy and cost-efficient solution for the resource allocation problem in heterogeneous network, and provided extensive analytical and experimental results to estimate the potential energy and cost savings that can be achieved. The studies in [21] and [22] further exploited small-cell sleeping potential and proposed small-cell control algorithms for power saving. The authors of [23] proposed a reverse auction based offloading that targets achieving tremendous energy efficiency under the constraints of QoS requirements. The optimization problem with constraints was solved by dynamic programming method with Karush-KuhnTucker (KKT) conditions. These approaches can find the optimal or sub-optimal decisions of small BSs modes under the constraint that the stochastic model is perfectly characterized and the needed information is accurate. For example, they assume that the network environment, including user location, service request and channel information, remains constant in the considered period. However, due to the stochastic nature in wireless heterogeneous networks, these assumptions can hardly be valid. In addition, these approaches usually have high computational complexities and need repetitive and intensive computation in each time slot. Moreover, non of the above schemes considers the exploitation of the cell system load to determine the optimal offloading strategy in the $5 \mathrm{G}$ heterogeneous environment and they do not emphasize load maintenance of small cells. The proposed scheme, however, not only considers the cell system load but also guarantees 
the QoS of the offloaded macro users traffic to small cells.

Machine learning is a powerful tool that penetrated the communication and networking field recently [24] [25] [26] [27]. It is envisioned as potential solution for efficient traffic offloading in heterogeneous cellular networks. Some of the machine learning based approaches such as reinforcement learning-based do not need a specific model or any non causal information. Instead, they can learn the environment model by interacting with the environment and refine their strategies accordingly. In [28], the authors formulated the traffic load variations in heterogeneous cellular networks as a Markov decision process. Then, they minimized the energy consumption of radio access networks, by designing a reinforcement learning-based framework to perform BS switching operation. The work in [29] proposed a deep reinforcement learningbased BS activation algorithm to obtain small cells activation decisions where small cells operation mode is formulated as a Markov decision process that operates in large time scale and utilizes statistics rather than user activity information. Non of the proposed machine learning-based traffic offloading schemes considers the awareness of macro BSs of each others' offloading decisions. This helps to obtain offloading decision that avoids congestion and interference at small cells as well as improves the offloading decision as it learns from other macro BSs traffic offloading strategies. To accomplish this, our proposed online reinforcement learning uses intuition feature in learning, which allows each macro BS to conjecture other macro BSs offloading strategies. This eliminates the overhead of information exchange between the macro BSs to achieve a cooperative traffic offloading strategy and it expedites the convergence of the proposed learning.

\section{SYSTEM DESCRIPTION}

This section describes the system model considered and the energy efficiency constrained problem formulation that maintains all cells system load within a predefined range.

\section{A. System Model}

The considered system model tackles the downlink communication in a multi-tier heterogeneous $5 \mathrm{G}$ network. The multi-tier structure consists of a primary tier represented by macrocells, and secondary tier that includes picocells and femtocells that share the same spectrum with the primary tier as in Figure 1. This multi-tier structure operates over discrete time slots each with constant time duration. The service region for each cell is represented by a set of $\mathbf{L}$ locations, each being characterized by uniform signal propagation conditions [30]. At each location $l \in \mathbf{L}$ in each time slot $(t=1,2, \ldots$,$) , the$ service requests follow Poisson arrival process with arrival rate $\lambda(l, t)$. The size of the requested traffic demand is assumed to be an exponentially distributed variable, which makes the network Markovian [31]. The coverage in the network is maintained by a set of macro BSs $\mathbf{K}=\{1,2, \ldots, K\}$ with $N_{k}$ small cells (pico and femto) that operate under the coverage of the macro BS $k \in \mathbf{K}$. The small BSs are connected to the macro BS using a logical interface. We choose $L_{k}$ and $L_{n}$ to designate the sets of locations covered by the macro
BS $k$ and small BS $n$ respectively, where $L_{n} \subset L_{k}$. The word cell and BS are used interchangeably in the rest of the paper. The macrocells are assumed to have an open access

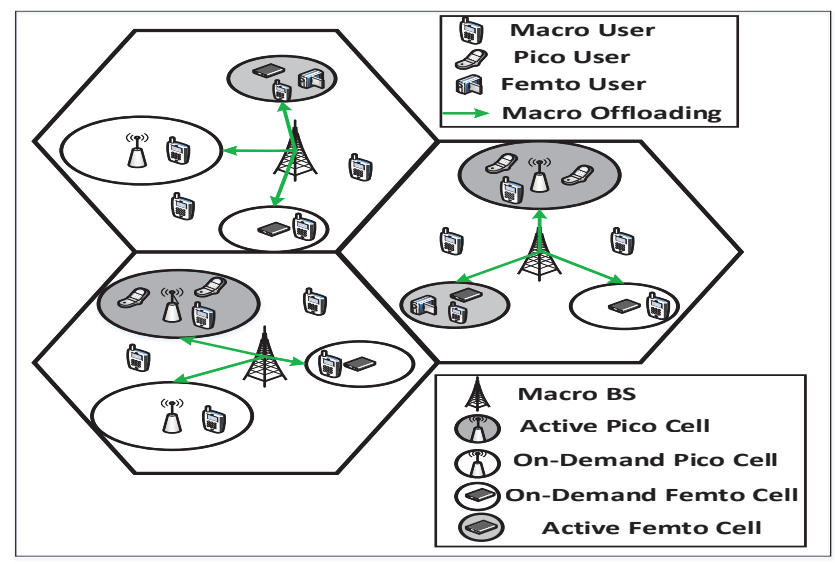

Figure 1. System Model

to small cells to offload their traffic to small cells whether they are already operative and serves small cells users or they will be activated on demand. The offloading decisions and the small cells operations mode selection are dynamically selected by the macro BS. If a small cell is activated and macro users are offloaded, the QoS requirements of the macro users within its coverage must be satisfied. In this model, we investigate traffic offloading problem from macrocell to small cells in an energy efficient fashion. The offloading process is triggered according to the system load of the macrocell. For example, when a macrocell is lightly loaded, the offloading is not necessary and small BSs maintain their state. However, the heavily loaded macrocells are allowed to offload traffic to activated small BSs and they remains operative to handle the traffic of the macro users that are not offloaded [32]. The macro BS controls the offloading process according to the number of small BSs within its coverage and their system load information. The cross-tier channel information is estimated at the macrocell and then a quantized version is forwarded to the corresponding small cell as in [33]. In order to reduce overhead cost of information exchange between macro BS and small BS, we adjust the information exchange rate according to the instantaneous channel conditions and it was demonstrated that if the exchange rate increases with the SNR then, there will be no performance degradation observed due to cross-tier interference.

Let $s(t)$ describes the evolution of the network state across time slots and it is defined as the number of users associated with different small BSs at different locations. Every small BS under the coverage of the macro BS $k \in \mathbf{K}$ is labeled as $k_{n}$ where $n=0,1,2, \ldots N_{k}, n=0$ for the macro BS and the location covered by the BS $k_{n}$ are labeled from 1 to $L_{k_{n}}$. Thus, the configuration of the macro BS and all small cells under its coverage is defined as follows,

$$
\begin{gathered}
s_{k}(t)=s_{k 0}^{1}(t), \ldots ., s_{k 0}^{L_{k 0}}(t), s_{k 1}^{1}(t), \ldots ., s_{k 1}^{L_{k 1}}(t), \\
\ldots, s_{k_{N_{k}}}^{1}(t), \ldots, s_{k_{N_{k}}}^{L_{k_{N_{k}}}}(t)
\end{gathered}
$$


Each element in (1) represents the number of users at the location covered by a small BS within the macro BS $k$ during time slot $t$. The operation mode for small BSs under the coverage of the macro $\mathrm{BS} k$ is represented by $A_{k}(t)=\left(a_{k 1}(t), \ldots, a_{k_{N_{k}}}(t)\right)$ with $a_{k_{n}}(t)=1$ if the small BS is selected for traffic offloading and 0 otherwise for all $n \in\left\{1, \ldots, N_{k}\right\}$. To proceed with the transmission scheduled to deliver the traffic demand of certain user, the system loads $S L_{k}(s, a)$ and $S L_{n}(s, a)$ for both macro and small cell respectively, during time slot $t$ are evaluated according to the traffic demand $\zeta(l, t)$ at certain time as

$$
\begin{aligned}
& S L_{k}(s, a)=\sum_{l \in L_{k}^{\prime}} \zeta(l, t) \times \frac{1}{T} \\
& S L_{n}(s, a)=\sum_{l \in L_{n}^{\prime}} \zeta(l, t) \times \frac{1}{T}
\end{aligned}
$$

where $L_{k}^{\prime}(t)=L_{k}$ excluding $L_{n}^{\prime}(t) \forall n \in \mathbf{N}$ and $L_{n}^{\prime}(t)$ are the set of locations associated with the macro BS $k$ and the small BS $n$ during the time slot $t$ respectively, and $T$ is the duration of the time slot in seconds. Note that $L_{n}^{\prime}(t)=L_{n} \subset L_{k}$ for an active small $\mathrm{BS}$ and $L_{n}^{\prime}(t)=\phi$ if the small cell is OFF. Consequently, the system load for each cell type can be interpreted as the fraction of time scheduled for serving the requested traffic demand or the probability of causing interference to other cells. The average signal to interference and noise ratio (SINR) achieved by the macro user (MUE) located at $l \in L_{k}$ associated with macro BS $k$ is evaluated as,

$\gamma_{k l}(s, a)=\frac{G_{k l} P_{k}}{\sum_{i \in \mathbf{K} /\{k\}} G_{i l} P_{i} S L_{i}(s, a)+\sum_{n \in \mathbf{N}} G_{n l} P_{n} S L_{n}(s, a)+\sigma}$

where $G_{k l}, G_{i l}$ and $G_{n l}$ are the channel gain between the macro user and the macro BS $k$, other macro BS $i$ and small BS $n$, respectively, $P_{k}, P_{i} P_{n}$ are the transmission power of macro BS $k$, other macro BS $i \in \mathbf{K}$ and small BS $n \in \mathbf{N}$ respectively, and $\sigma$ is the background noise. The SINR for the offloaded macro user associated with small BS $n$ located at $l \in L_{n}$ is calculated as follows,

$\gamma_{n l}(s, a)=\frac{G_{n l} P_{n}}{\sum_{k \in \mathbf{K}} G_{k l} P_{k} S L_{k}(s, a)+\sum_{q \in \mathbf{N} /\{n\}} G_{q l} P_{q} S L_{q}(s, a)+\sigma}$

where $G_{n l}, G_{k l}$ and $G_{q l}$ are the channel gain between the offloaded users and the serving small BS $n$, macro BS $k$ and other small BS $q$, respectively at location $l, P_{n}, P_{k} P_{q}$ are the transmission power of the serving small BS $n$, macro BS $k \in \mathbf{K}$ and other small BS $q \in \mathbf{N}$ respectively. The achievable data rates for MUEs associated with the macro BS and MUEs offloaded to small BS are calculated as in (6) and (7) respectively,

$$
\begin{aligned}
& R_{k l}(s, a)=B \log _{2}\left(1+\gamma_{k l}(s, a)\right) \\
& R_{n l}(s, a)=B \log _{2}\left(1+\gamma_{n l}(s, a)\right)
\end{aligned}
$$

where $B$ is the system bandwidth.

\section{B. Problem Formulation}

In this section, we formulate the energy aware traffic offloading in $5 \mathrm{G}$ heterogeneous network, which is described by users arrival, generation of service requests, BSs that serve these requests, and system load for each cell. Heavily loaded macro BSs provide poor QoS, which require traffic offloading to small cells that are within the coverage of the heavily loaded macrocell. The energy efficiency achieved by certain macrocell or small cell is dependent on the system load of that cell. Therefore, for certain network configuration $s(t)$ and offloading decision $a(t)$ at time slot $t$, the energy efficiency of macro user associated with macro BS $k \in \mathbf{K}$ is calculated as follows,

$$
E E_{k}(s, a)=\frac{R_{k l}(s, a)}{P_{c t}^{k}+\Gamma_{k} S L_{k}(s, a) P_{k}}
$$

where $P_{c t}^{k}$ is the power consumed by the BS circuit for signal processing and $\Gamma_{k}$ is a linear transmission power dependence factor. Similarly, the energy efficiency for macro user associated with small cell $n$ is calculated as follows,

$$
E E_{n}(s, a)=\frac{R_{n l}(s, a)}{P_{c t}^{n}+\Gamma_{n} S L_{n}(s, a) P_{n}} \text { as } a_{k_{n}}(t)=1
$$

where $P_{c t}^{n}$ is the power consumed by the BS circuit for signal processing and $\Gamma_{n}$ is a linear transmission power dependence factor. The condition $a_{k_{n}}(t)=1$ to indicate that energy efficiency only covers the small cells that receive offloaded traffic. The total energy efficiency for the entire network in time slot $t$ is found as,

$$
E E_{c}(s, a)=\sum_{k \in \mathbf{K}}\left(E E_{k}(s, a)+\sum_{n \in \mathbf{N}} E E_{n}(s, a)\right)
$$

The system load $S L_{z}(s, a)$, where $z \in \mathbf{K} \cup \mathbf{N}$ and $t=1,2,3 \ldots$, is utilized to quantify energy efficiency as it grows with the traffic demand and the interference from the active cells. In addition, the user QoS represented by throughput or delay is also dependent on the system load as when the system load is low, BSs are able to offer their users a good capacity to meet QoS requirements. However, heavily loaded system leads to poor QoS and service outage. The optimization problem for traffic offloading strategy that maximizes the netwrok energy $\sigma$ efficiency is formulated as follows,

$$
\begin{gathered}
\max _{\pi \in \Pi} E E_{c}(\pi(s, a)) \text { s.t } \\
C .1 S L_{z}(s, a) \leq S L_{z}^{t h} \\
C .2 R_{u} \geq R_{u}^{t h}
\end{gathered}
$$

where $\Pi$ is the set of all available traffic offloading strategies, and $S L_{z}^{t h}$ is a predefined threshold for the system load for each cell. The constraint in C.1 indicates that the selected offloading strategy should maintain the cell system load below certain threshold. The users application QoS is also maintained above certain threshold for macro user $u$ whether it is still associated with the macro BS or it is offloaded as in C.2. Note that when the threshold's value is small, the BS operates with low usage of resources and experience high throughput and less delay. 


\section{TRAFFIC OfFloAding USING InTUitive ONLINE REINFORCEMENT LEARNING}

The goal of this section is to tackle the offloading optimization problem formulated in section III.B using our developed intuitive online reinforcement learning. We define the learning model that is associated to each network state, action, transition function and reward. Then, our proposed intuitive learning mechanism for traffic offloading in $5 \mathrm{G}$ networks is described.

\section{A. Online Reinforcement Learning Model}

In this model, each macro BS plays the role of an intelligent agent, which observes the network state $s(t)$ and its associated action $a(t)$ in the current time slot $(t)$. At the end of each time slot, the reward, which evaluates the selected action is generated and a state transition to the next state is performed. The state, action, transition function and reward for our model are defined as follows,

- State: Since there is no cooperation among the macrocells, the network state $s(t)$ is defined based on the local information about the configuration of BSs and their coverage locations defined in (1).

- Action: The action selected by the macro BS $a(t)$ according to the state information is defined as the offloading decision to certain small $\mathrm{BS}$, which takes the value $a_{k_{n}}(t)=1$ if the small BS is selected for offloading and 0 otherwise.

- Reward: The reward function is the received reward due to the selected action and is defined as as $R W(s, a)=$ $E E_{c}(s, a)$ where $E E_{c}(s, a)$ is the energy efficiency defined in (10). However, the reward function is only achievable if the constraints $C .1$ and $C .2$ are met. Otherwise, it is evaluated to 0 .

- Transition Function: The transition function $T_{f}$ is evaluated while moving from the state $s(t)$ to $s^{\prime}(t+1)$ as a result of the selected offloading action $a_{k_{n}}(t)$ by the macro BS at time slot $t$. The transition function is defined according to a transition probability given as follows,

$$
T_{f}\left(s, a, s^{\prime}\right)=\operatorname{Pr}\left(s(t+1)=s^{\prime} \mid s(t)=s, a(t)=a\right)
$$

This transition probability is evaluated according to [34] and it depends on the arrival of the users in the network.

In non-cooperative online reinforcement learning [35], each macrocell $k \in \mathbf{K}$ selects the strategy $\pi_{k}\left(s_{k}\right)$ independently to maximize its total discounted reward. However, independent learning is subjective to the interference and congestion problem at the small cells as the macrocells are not aware of each other's traffic offloading strategies. Therefore, it is necessary that each macro BS becomes aware of other macro BSs traffic offloading strategies and learn cooperatively to reach the most appropriate offloading strategy and avoid interference and congestion at small cells. This is achieved by defining a common goal of finding a joint traffic offloading strategy $\pi \in \Pi$ that maximizes the total energy efficiency with consideration of other macro BSs offloading strategies. Thus, the total discounted reward in our model is defined as follows,

$$
\max _{\pi_{k} \in \Pi_{k}}\left\{E\left[\sum_{t=0}^{\infty} \beta^{t} R W_{k}\left(s_{k}^{t}, \pi_{k}\left(s_{k}^{t}\right), \pi_{-k}\left(s_{k}^{t}\right)\right)\right]\right\}
$$

where $\pi_{-k}\left(s_{k}^{t}\right)=\left(\pi_{1}\left(s_{1}^{t}\right), \ldots ., \pi_{k-1}\left(s_{k-1}^{t}\right), \pi_{k+1}\left(s_{k+1}^{t}\right), \ldots\right.$. , $\left.\pi_{K}\left(s_{K}^{t}\right)\right)$ and $\beta$ is the discount factor. The strategy $\pi_{k}\left(s_{k}, a_{k}\right)$ is defined as the probability vector with which the macro BS $k$ selects action $a_{k}=a_{k_{n}}(t)$ at the state $s_{k}$. and $-k$ is the index for other macro BSs. The total expected discounted reward of cell $k$ over infinite iterations and with being aware of other cells strategies $\pi_{-k}$ is given by,

$$
\begin{gathered}
V_{k}\left(s_{k}, \pi_{k}, \pi_{-k}\right)=E\left[R W_{k}\left(s_{k}, \pi_{k}\left(s_{k}\right), \pi_{-k}\left(s_{k}\right)\right)\right]+ \\
\left.\beta \sum_{s_{k}^{\prime} \in S_{k}} T_{f}\left(s_{k}, a, s_{k}^{\prime}\right)\left(\pi_{k}\left(s_{k}\right)\right), \pi_{-k}\left(s_{k}\right)\right) V_{k}\left(s_{k}^{\prime}, \pi_{k}, \pi_{-k}\right)
\end{gathered}
$$

where,

$$
\begin{gathered}
E\left[R W_{k}\left(s_{k}, \pi_{k}\left(s_{k}\right), \pi_{-k}\left(s_{k}\right)\right)\right]= \\
\sum_{\left(a_{k}, a_{-k}\right) \in A}\left[R W_{k}\left(s_{k}, a_{k}, a_{-k}\right) \prod_{j \in \mathbf{K} /\{k\}} \pi_{j}\left(s_{j}, a_{j}\right)\right]
\end{gathered}
$$

The best strategy that the learning agent aims to achieve $\pi_{k}^{*}$ for each environment state and satisfies Bellman's optimality equation [36], achieves the following total discounted reward,

$$
\begin{gathered}
V_{k}\left(s_{k}, \pi_{k}^{*}, \pi_{-k}^{*}\right)=\max _{a_{k} \in A_{k}}\left\{E\left[R W_{k}\left(s_{k}, a_{k}, \pi_{-k}^{*}\left(s_{k}\right)\right)\right]+\right. \\
\beta \sum_{s_{k}^{\prime} \in S_{k}} T_{s_{k}, s_{k}^{\prime}}\left(a_{k}, \pi_{-k}\left(s_{k}\right) V_{k}\left(s_{k}^{\prime}, \pi_{k}^{*}, \pi_{-k}^{*}\right)\right.
\end{gathered}
$$

where

$$
\begin{gathered}
E\left[R W_{k}\left(s_{k}, a_{k}, \pi_{-k}^{*}\left(s_{k}\right)\right)\right]= \\
\sum_{\left(a_{k}, a_{-k}\right) \in A}\left[R W_{k}\left(s_{k}, a_{k}, a_{-k}\right) \prod_{j \in \mathbf{K} /\{k\}} \pi_{j}^{*}\left(s_{j}, a_{j}\right)\right]
\end{gathered}
$$

We define the optimal Q-value $Q_{k}^{*}\left(s_{k}, a_{k}\right)$ of the macro BS $k$ as the sum of the current reward and the future expected rewards when all the macrocells achieve their best offloading strategy as follows,

$$
\begin{gathered}
Q_{k}^{*}\left(s_{k}, a_{k}\right)=E\left[R W_{k}\left(s_{k}, a_{k}, \pi_{-k}^{*}\left(s_{k}\right)\right)\right]+\beta \\
\sum_{s_{k}^{\prime} \in S_{k}} T_{s_{k}, s_{k}^{\prime}}\left(a_{k}, \pi_{-k}^{*}\left(s_{k}\right)\right) V_{k}\left(s_{k}^{\prime}, \pi_{k}^{*}, \pi_{-k}^{*}\right)= \\
E\left[R W_{k}\left(s_{k}, a_{k}, \pi_{-k}^{*}\left(s_{k}\right)\right)\right]+ \\
\beta \sum_{s_{k}^{\prime} \in S_{k}} T_{s_{k}, s_{k}^{\prime}}\left(a_{k}, \pi_{-k}^{*}\left(s_{k}\right)\right) \max _{b_{k} \in A_{k}} Q_{k}^{*}\left(s_{k}^{\prime}, b_{k}\right)
\end{gathered}
$$

The optimal Q-value is reached in a recursive way using $\left(a_{k}, s_{k}, s_{k}^{\prime}, \pi_{k}^{t}\right)$, where $s_{k}=s_{k}^{t}$ and $s_{k}^{\prime}=s_{k}^{t+1}$ are the environment states observed by the macro BS $k$ at iteration $t$ and $t+1$ respectively. Thus, the general online learning updating rule is defined as,

$$
\begin{gathered}
Q_{k}^{t+1}\left(s_{k}, a_{k}\right)=\left(1-\alpha^{t}\right) Q_{k}^{t}\left(s_{k}, a_{k}\right)+ \\
\alpha^{t}\left\{\sum_{a_{-k} \in A_{-k}}\left[R W_{k}\left(s_{k}, a_{k}, a_{-k}\right) \prod_{j \in \mathbf{K} /\{k\}} \pi_{j}^{t}\left(s_{j}, a_{j}\right)\right]\right\}
\end{gathered}
$$




$$
\left.+\beta \max _{b_{k} \in A_{k}} Q_{k}^{t}\left(s_{k}^{\prime}, b_{k}\right)\right\}
$$

where $\alpha^{t} \in[0,1)$ is the learning rate. The $\mathrm{Q}$-value increases when the macrocell $k$ achieves higher reward by combining the old value and the future expected reward.

\section{B. Intuitive Online Reinforcement Learning Mechanism for Traffic Offloading}

From the online reinforcement learning model described in the IV.A, we notice that the reward achieved by each macrocell is a function of the offloading strategies of other macrocells. Accordingly, our proposed model is a multi-agent online reinforcement learning that benefits from the awareness about other macrocells offloading strategies and improves the quality of the selected offloading action. However, the offloading problem is challenging in this context as information about the other macrocells and their offloading strategies are unavailable. Moreover, exchanging information about these strategies between the macro BSs requires large computation and causes considerable overhead. Local information like environment state, traffic offloading strategy and the received historical rewards is the only information available. Thus, we present our intuitive online learning mechanism that is able to conjecture the offloading strategies of the other macrocells and exploits this information to reach the best offloading strategy. Our mechanism relies on the fact that each macro BS is aware of the system loads, activities and coverage locations of all the small BSs operate under its coverage.

We define $\mu^{t}\left(s_{k}, a_{-k}\right)=\prod_{j \in \mathbf{K} /\{k\}} \pi_{j}^{t}\left(s_{j}, a_{j}\right)$ for macrocell $k$ in time slot $t$, to be the intuition factor used to determine $Q_{k}^{t+1}\left(s_{k}, a_{k}\right)$ value in the next iteration when all the macrocells select actions $a_{-k}$ according to their corresponding offloading strategies $\pi_{-k}^{t}\left(s_{-k}\right)$. The macrocell determines the intuition factor $\mu$ from the local observations. The intuition idea is derived from the concept that different macrocells follow similar traffic offloading strategies at the same network states. The probability of experiencing certain environment state $s_{k}^{t}$ and achieve certain reward $R W_{k}$ is defined as $p t=$ $\pi^{t}\left(s_{k}, a_{k}\right) \mu^{t}\left(s_{k}, a_{-k}\right)$. Let $v$ denotes the number of iterations between any two consecutive iterations in which macrocell $k$ achieves the same reward $R W_{k}\left(s_{k}, a_{k}, a_{-k}\right)$, then $v$ has independent and identical distribution (i.i.d) with probability $p t$. Consequently, $p t=\frac{1}{1+v^{\prime}}$, where $v^{\prime}$ is the mean value of $v$ and is calculated through observations of local reward history. Therefore, the intuition factor can be estimated from the local historical information about offloading strategies using linear model as follows,

$$
\mu_{k}^{h}\left(s_{k}, a_{-k}\right)=\mu_{k}^{\prime}\left(s_{k}, a_{-k}\right)-w_{k}\left[\pi_{k}^{t}\left(s_{k}, a_{k}\right)-\pi_{k}^{\prime}\left(s_{k}, a_{k}\right)\right]
$$

where $\mu_{k}^{\prime}\left(s_{k}, a_{-k}\right)$ and $\pi_{k}^{\prime}\left(s_{k}, a_{k}\right)$ are the reference points for specific intuition factor and strategy selection probability respectively. These reference points are given from common knowledge provided that each macrocell $k$ assumes that other macrocells observe its deviation from its reference points by a quantity proportional to the deviation of $\pi_{i}^{t}\left(s_{k}, a_{k}\right)-$ $\pi_{k}^{\prime}\left(s_{k}, a_{k}\right)$. The macrocells are assumed to update these reference points based on their local observation. At time slot $t$, the macro BS $k$ sets $\mu_{k}^{\prime}\left(s_{k}, a_{-k}\right)$ and $\pi_{k}^{\prime}\left(s_{k}, a_{k}\right)$ to be $\mu_{i}^{t-1}\left(s_{k}, a_{-k}\right)$ and $\pi_{k}^{t-1}\left(s_{k}, a_{k}\right)$ respectively. As a result, the intuition factor will be,

$\mu_{k}^{h}\left(s_{k}, a_{-k}\right)=\mu_{k}^{t-1}\left(s_{k}, a_{-k}\right)-w_{k}\left[\pi_{k}^{t}\left(s_{k}, a_{k}\right)-\pi_{k}^{t-1}\left(s_{k}, a_{k}\right)\right]$

The definition in (21) comes from the fact that if the macro BS $k$ changes its strategy, this will induce other macro BSs to perform changes in their offloading strategies in the next time slot. Now, every macro BS can conjecture other BSs strategies using the intuition factor. Therefore, the updating rule in (19) is modified in away that macro BS $k$ updates its Q-value with its own information during the online reinforcement learning as follows,

$$
\begin{gathered}
Q_{k}^{t+1}\left(s_{k}, a_{k}\right)=\left(1-\alpha^{t}\right) Q_{k}^{t}\left(s_{k}, a_{k}\right)+\alpha^{t}\{ \\
\left.\sum_{a_{-k} \in A_{-k}} \mu_{k}^{h}\left(s_{k}, a_{-k}\right) R W_{k}\left(s_{k}, a_{k}, a_{-k}\right)+\beta \max _{b_{k} \in A_{k}} Q_{k}^{t}\left(s_{k}^{\prime}, b_{k}\right)\right\}
\end{gathered}
$$

The exploration vs exploitation balancing is important issue in online reinforcement learning. Exploration aims to try new offloading strategies while exploitation seizes the already explored strategies with positive feedback. To balance exploration vs exploitation and avoid the problem of choosing equally among actions in the exploration process as in the $\epsilon$ greedy selection [37], actions are selected according to graded functions of their Q-value. For example, the offloading strategy with the highest Q-value has the highest selection probability. We exploit Boltzman distribution [35] to perform this selection where the macro BS $k$ selects an action $a_{k}$ in state $s_{k}$ at time slot $t$ with the following probability,

$$
\pi_{k}^{t}\left(s_{k}, a_{k}\right)=\frac{e^{Q_{k}^{t}\left(s_{k}, a_{k}\right)} / \tau}{\sum_{b_{k} \in A_{k}} e^{Q_{k}^{t}\left(s_{k}, b_{k}\right)} / \tau}
$$

where $\tau$ is a positive parameter called the temperature. High temperature causes the action probabilities to be all nearly equal, while low temperature induces a large difference in selection probabilities for actions differ in their Q-values. The intuition based traffic offloading algorithm executed by the macro BSs is presented in Algorithm 1.

The algorithm starts by checking the state information at the macrocell including its system load. If the macrocell is overloaded, this triggers the need for offloading traffic to small cells under its coverage. The macrocell searches for small cells for traffic offloading based on their coverage locations. Then, the intuitive learning traffic offloading process is initialized. The Q-values for each strategy in different states are evaluated. When the state is initialized, offloading action is selected. After the action of the selected strategy is applied, the system load of the active small cell (condition C.1) is checked to ensure that the cells are not overloaded and the reward function is evaluated only if the system load is below its corresponding threshold and macro users data rate is above its corresponding threshold. At the end, the Q-value, intuition factor, and strategy are updated accordingly. The process is repeated until the system converges to the best traffic offloading strategy. 


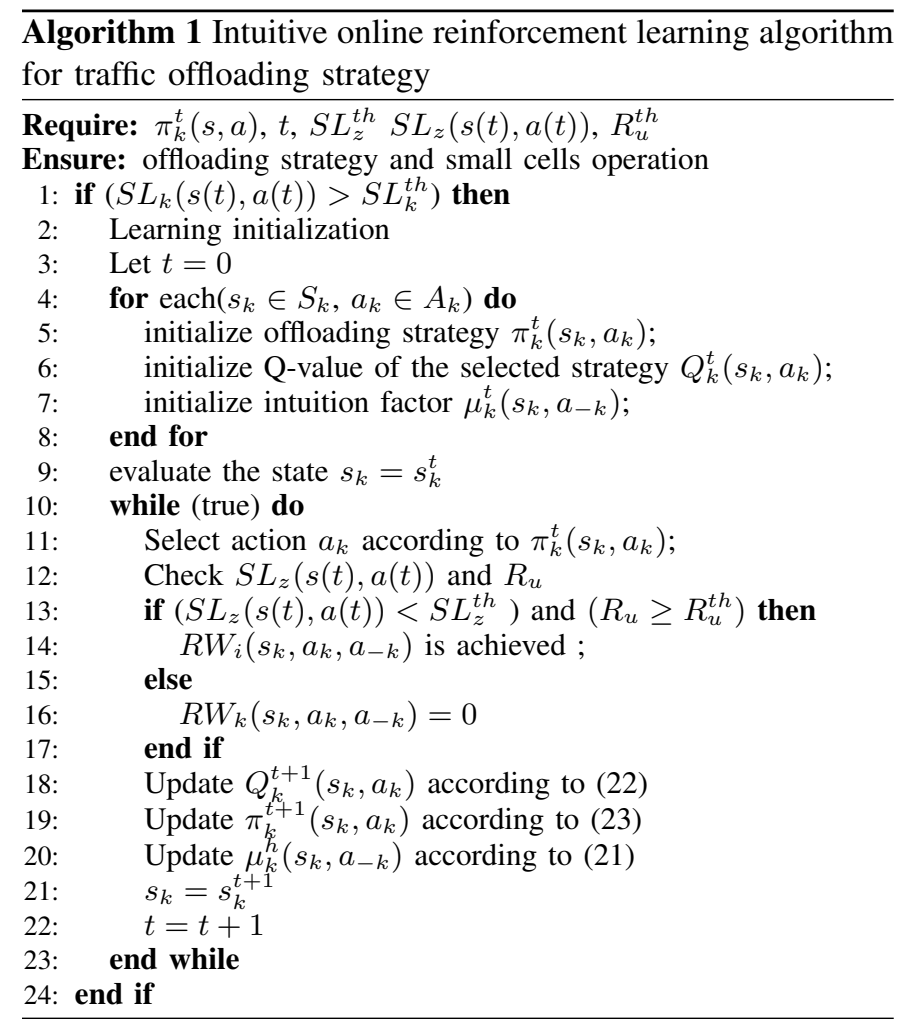

\section{Intuitive Online Reinforcement Learning Convergence}

The proposed intuitive online learning algorithm converges with probability 1 if the conditions in Lemma 1 proposed in [36], which creates the convergence conditions of general online reinforcement learning process are satisfied, given that $\mathcal{H}$ is a pseudo-contraction operator and $\Re$ is the space for all Q-values.

Lemma 1. Assume that the learning rate $\alpha^{t}$ in (22) satisfies the sufficient conditions of Theorem in [36], and the mapping $\mathcal{H}^{t}: \Re \rightarrow \Re$ meets the following condition: there exists a number $0<\beta<1$ and a sequence $\varphi \geq 0$ converging to zero w.p. 1, such that $\left\|\mathcal{H}^{t} Q^{t}-\mathcal{H}^{t} Q^{*}\right\| \leq \beta\left\|Q^{t}-Q^{*}\right\|+\varphi^{t}$ for all $Q^{t} \in \Re$ and $Q^{*}=E\left[\mathcal{H}^{t} Q^{*}\right]$, then the iteration defined by,

$$
Q^{t+1}=\left(1-\alpha^{t}\right) Q^{t}+\alpha^{t}\left(\mathcal{H}^{t} Q^{t}\right)
$$

converges to $Q^{*}$ w.p. 1 .

We define the mapping operator $\mathcal{H}$ as a mapping on a complete metric space $\Re \rightarrow \Re$, where,

$$
\begin{aligned}
\mathcal{H}^{t} Q_{k}^{t}\left(s_{k}, a_{k}\right)= & \sum_{a_{-k} \in A_{-k}} \mu_{k}^{h}\left(s_{k}, a_{-k}\right) R W_{k}\left(s_{k}, a_{k}, a_{-k}\right) \\
& +\beta \max _{b_{k} \in A_{k}} Q_{k}^{t}\left(s_{k}^{\prime}, b_{k}\right)
\end{aligned}
$$

Now, we proceed to prove the two conditions in Lemma 1 with the mapping operator defined in (24).

Proposition 1. For K-agent stochastic learning,

$$
Q^{*}=E\left[\mathcal{H}^{t} Q^{*}\right]
$$

where $Q^{*}=\left(Q_{1}^{*}, \ldots, Q_{K}^{*}\right)$
Proof: As

$$
\begin{gathered}
Q_{k}^{*}\left(s_{k}, a_{k}\right)=E\left[R W_{k}\left(s_{k}, a_{k}, \pi_{-k}^{*}\left(s_{k}\right)\right)\right]+ \\
\beta \sum_{s_{k}^{\prime} \in S_{k}} T_{s_{k}, s_{k}^{\prime}}\left(a_{k}, \pi_{-k}^{*}\left(s_{k}\right)\right) \max _{b_{k} \in A_{k}} Q_{k}^{*}\left(s_{k}^{\prime}, b_{k}\right) \\
=\sum_{s_{k}^{\prime} \in S_{k}} T_{s_{k}, s_{k}^{\prime}}\left(a_{k}, \pi_{-k}^{*}\left(s_{k}\right)\right)\left\{\sum_{a_{-k} \in A_{-k}} R W_{k}\left(s_{k}, a_{k}, a_{-k}\right)\right. \\
\left.\prod_{j \in \mathbf{K} /\{k\}} \pi_{j}^{*}\left(s_{j}, a_{j}\right)+\beta \max _{b_{k} \in A_{k}} Q_{k}^{*}\left(s_{k}^{\prime}, b_{k}\right)\right\}
\end{gathered}
$$

With optimal intuition factor defined in Section IV.B as $\mu_{k}^{*}\left(s_{k}, a_{-k}\right)=\prod_{j \in \mathbf{K} /\{k\}} \pi_{j}^{*}\left(s_{j}, a_{j}\right)$. Thus,

$$
Q_{k}^{*}\left(s_{k}, a_{k}\right)=E\left[\mathcal{H}^{t} Q_{k}^{*}\left(s_{k}, a_{k}\right)\right]
$$

for all $s_{k} \in S_{k}$ and $a_{k} \in A_{k}$

To prove the second condition in Lemma 1, we need to define the distance between any two Q-values $Q$ and $Q^{\prime} \in \Re$ as follows,

$$
\left\|Q-Q^{\prime}\right\|=\max _{k \in \mathbf{K}} \max _{s_{k} \in S_{k}} \max _{a_{k} \in A_{k}}\left|Q_{k}\left(s_{k}, a_{k}\right)-Q^{\prime}\left(s_{k}, a_{k}\right)\right|
$$

Proposition 2. $\mathcal{H}^{t}$ is a contraction mapping operator in online reinforcement learning

$$
\begin{aligned}
& \text { Proof: } \quad\left\|\mathcal{H}^{t} Q-\mathcal{H}^{t} Q^{\prime}\right\|= \\
& \max _{k \in \mathbf{K}} \max _{s_{k} \in S_{k}} \max _{a_{k} \in A_{k}}\left|\mathcal{H}^{t} Q_{k}\left(s_{k}, a_{k}\right)-\mathcal{H}^{t} Q^{\prime}\left(s_{k}, a_{k}\right)\right|
\end{aligned}
$$

Then, we substitute the value of $\mathcal{H}^{t} Q_{k}\left(s_{k}, a_{k}\right)$ from (24),

$$
\begin{gathered}
\mid\left\|\mathcal{H}^{t} Q-\mathcal{H}^{t} Q^{\prime}\right\|= \\
\max _{k \in \mathbf{K}} \max _{s_{k} \in S_{k}} \max _{a_{k} \in A_{k}} \mid \sum_{a_{-k} \in A_{-k}}\left[\mu_{k}^{\prime \prime}\left(s_{k}, a_{-k}\right)-\mu_{k}^{;}\left(s_{k}, a_{-k}\right)\right] \\
\times R W_{k}\left(s_{k}, a_{k}, a_{-k}\right)+\beta\left[\max _{b_{k} \in A_{k}} Q_{k}\left(s_{k}^{\prime}, b_{k}\right)-\max _{b_{k} \in A_{k}} Q_{k}^{\prime}\left(s_{k}^{\prime}, b_{k}\right)\right] \mid \\
\leq \max _{k \in \mathbf{K}} \max _{s_{k} \in S_{k}} \max _{a_{k} \in A_{k}} \mid \sum_{a_{-k} \in A_{-k}}\left[\mu_{k}^{\prime \prime}\left(s_{k}, a_{-k}\right)-\mu_{k}^{;}\left(s_{k}, a_{-k}\right)\right] \times \\
R W_{k}\left(s_{k}, a_{k}, a_{-k}\right)\left|+\max _{k \in \mathbf{K}} \max _{b_{k} \in A_{k}} \beta\right| Q_{k}\left(s_{k}^{\prime}, b_{k}\right)-Q_{k}^{\prime}\left(s_{k}^{\prime}, b_{k}\right) \mid \\
\leq \max _{k \in \mathbf{K}} \max _{s_{k} \in S_{k}} \max _{a_{k} \in A_{k}} \mid \sum_{a_{-k} \in A_{-k}}\left[\mu_{k}^{\prime \prime}\left(s_{k}, a_{-k}\right)-\mu_{k}^{;}\left(s_{k}, a_{-k}\right)\right] \times \\
R W_{k}\left(s_{k}, a_{k}, a_{-k}\right) \mid+\beta\left\|Q-Q^{\prime}\right\|
\end{gathered}
$$

We can use the definition of intuition factor and reference points in (20) to get,

$$
\begin{aligned}
& \sum_{a_{-k} \in A_{-k}}\left[\mu_{k}^{\prime}\left(s_{k}, a_{-k}\right)-\mu_{k}^{;}\left(s_{k}, a_{-k}\right)\right] R W_{k}\left(s_{k}, a_{k}, a_{-k}\right) \\
= & -\sum_{a_{-k} \in A_{-k}} w_{k}\left[\pi_{k}\left(s_{k}, a_{k}\right)-\pi_{k}^{\prime}\left(s_{k}, a_{k}\right)\right] R W_{k}\left(s_{k}, a_{k}, a_{-k}\right)
\end{aligned}
$$

The definition of $\pi_{k}\left(s_{k}, a_{k}\right)$ is borrowed from (23) with assumption of large $\tau$ as follows,

$$
e^{Q_{k}\left(s_{k}, a_{k}\right) / \tau}=1+\frac{Q_{k}\left(s_{k}, a_{k}\right)}{\tau}+\psi\left(\frac{Q_{k}\left(s_{k}, a_{k}\right)}{\tau}\right)
$$


where $\psi$ is a polynomial of order $O\left(\left(\frac{Q_{k}\left(s_{k}, a_{k}\right)}{\tau}\right)^{2}\right)$. Thus,

$\sum_{b \in A_{k}} e^{Q_{k}\left(s_{k}, a_{k}\right) / \tau}=a_{k}+1+\sum_{b \in A_{k}}\left[\frac{Q_{k}\left(s_{k}, b\right)}{\tau}+\psi\left(\frac{Q_{k}\left(s_{k}, b\right)}{\tau}\right)\right]$

It can be proved that,

$\pi_{i}\left(s_{k}, a_{k}\right)=\frac{1}{a_{k}+1}+\frac{1}{a_{k}+1}+\frac{Q_{k}\left(s_{k}, a_{k}\right)}{\tau}+\eta\left(\frac{Q_{k}\left(s_{k}, b\right)}{\tau}\right)_{b}$

where $\eta\left(\frac{Q_{k}\left(s_{k}, b\right)}{\tau}\right)_{b}$ is a polynomial of order smaller than $O\left(\frac{Q_{k}\left(s_{k}, a_{k}\right)}{\tau}\right)_{b}$. Similarly,

$\pi_{k}^{\prime}\left(s_{k}, a_{k}\right)=\frac{1}{a_{k}+1}+\frac{1}{a_{k}+1}+\frac{Q_{k}^{\prime}\left(s_{k}, a_{k}\right)}{\tau}+\eta\left(\frac{Q_{k}^{\prime}\left(s_{k}, b\right)}{\tau}\right)_{b}$

Substituting (27) and (28) in (26), we get,

$$
\begin{gathered}
\sum_{a_{-k} \in A_{-k}}\left[\mu_{k}^{\prime \prime}\left(s_{k}, a_{-k}\right)-\mu_{k}^{i}\left(s_{k}, a_{-k}\right)\right] R W_{k}\left(s_{k}, a_{k}, a_{-k}\right)= \\
-\frac{\sum_{a_{-k} \in A_{-k}} w_{k} R W_{k}\left(s_{k}, a_{k}, a_{-k}\right)}{\tau} \times
\end{gathered}
$$

$\frac{1}{a_{k}+1}\left[Q_{k}\left(s_{k}, a_{k}\right)-Q_{k}^{\prime}\left(s_{k}, a_{k}\right)\right]+\eta\left(\frac{Q_{k}\left(s_{k}, b\right)}{\tau}\right)_{b}-\eta\left(\frac{Q_{k}^{\prime}\left(s_{k}, b\right)}{\tau}\right)_{b}$

With large $\tau$ assumption,

$$
\begin{gathered}
\left|\sum_{a_{-k} \in A_{-k}}\left[\mu_{k}^{\prime \prime}\left(s_{k}, a_{-k}\right)-\mu_{k}^{;}\left(s_{k}, a_{-k}\right)\right] R W_{k}\left(s_{k}, a_{k}, a_{-k}\right)\right| \\
\leq \frac{1-\beta}{a_{k}+1}\left|Q_{k}\left(s_{k}, a_{k}\right)-Q_{k}^{\prime}\left(s_{k}, a_{k}\right)\right|
\end{gathered}
$$

This brings us to the conclusion that,

$$
\begin{gathered}
\left\|\mathcal{H}^{t} Q-\mathcal{H}^{t} Q^{\prime}\right\| \leq \max _{k \in \mathbf{K}} \max _{s_{k} \in S_{k}} \max _{a_{k} \in A_{k}} \frac{1-\beta}{a_{k}+1} \\
\left|Q_{k}\left(s_{k}, a_{k}\right)-Q_{k}^{\prime}\left(s_{k}, a_{k}\right)\right|+\beta\left\|Q-Q^{\prime}\right\| \\
\leq \frac{1-\beta}{a+1}\left\|Q-Q^{\prime}\right\|+\beta\left\|Q-Q^{\prime}\right\| \leq \frac{\beta a+1}{a+1}\left\|Q-Q^{\prime}\right\|
\end{gathered}
$$

where $a=\min _{k \in \mathbf{K}} a_{k}$, therefore,

$$
\frac{\beta a+1}{a+1}<1
$$

This implies that $\mathcal{H}^{t}$ is a contraction mapping operator.

We clearly notice that we applied Lemma 1 to our intuition based learning model in (22) and prove its convergence by satisfying the conditions of Lemma 1 through Proposition 1 and Proposition 2 provided that $\tau$ is large enough.

\section{Numerical Results}

We simulate our proposed traffic offloading scheme to demonstrate its capability to boost the energy efficiency and maintain QoS for macro users in the system. The simulation environment is a two-tier $5 \mathrm{G}$ heterogeneous network, which is composed of 4 macrocells, 7 picocells, and 10 femtocells in a $3 \times 3 \mathrm{~km}^{2}$ square area as in Figure 2. The macro BSs are located at equal distance apart while the other cells are distributed randomly within macrocells coverage. The radius of the macrocell, picocells and femtocells are $\sqrt{2} / 2 \mathrm{~km}, 0.2$ $\mathrm{km}$, and $0.1 \mathrm{~km}$ respectively. The entire area is divided into
3600 small locations, where each location accounts for a small area with a resolution of $50 \times 50 \mathrm{~m}^{2}$. The channel gains are fixed as $G_{n l}=d s_{n l}^{-\epsilon}$ for all $n \in \mathbf{K} \cup \mathbf{N}$ and $l \in L$, where $d s_{n l}$ is the physical distance between BS $n$ and the center of location $l$ and $\epsilon$ is the path loss factor. Table I presents the values for other simulation parameters. Each simulation time is supposed to be 40 second to avoid switching small BSs including pico and femto BSs ON and OFF. The coverage

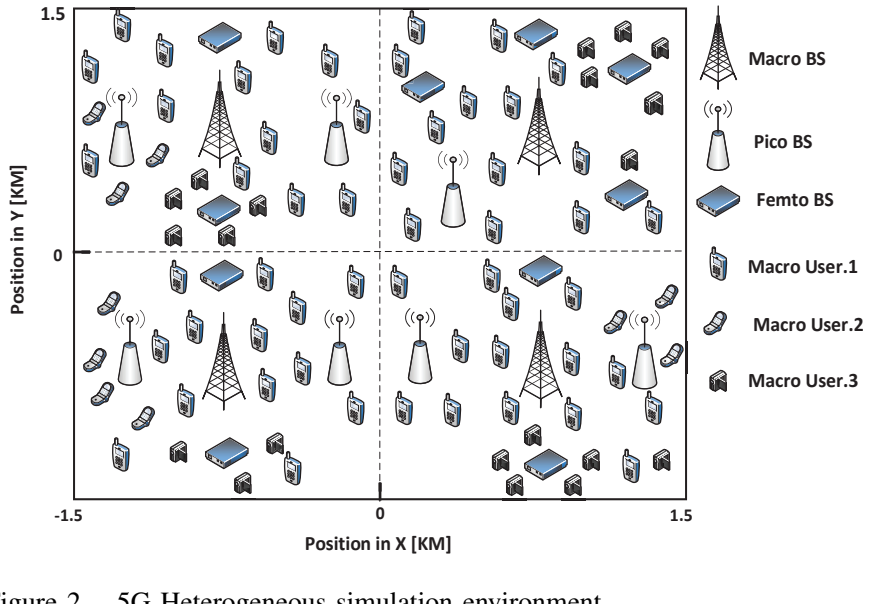

Figure 2. 5G Heterogeneous simulation environment

locations are assumed to be heavily loaded with an arrival rate of $\lambda=6 \lambda_{0}$ where $\lambda_{0}=0.3$ users/time slot is the arrival rate of macro users. We assume a predefined threshold for the system load to balance energy efficiency and QoS tradeoff, while the data rate threshold varies according to the type of BS that the user is associated with.

We compare the performance of our proposed traffic offloading scheme with the schemes proposed in [38], [39] and [40] in addition to the standard, where there is no offloading mechanism implemented. The work proposed in [38] exploits linear programing to develop a heuristic based offloading algorithm. The scheme proposed in [39] called (JPUA) proposed a mechanism with joint macro BS transmit power control and load-aware user association to reduce the interference impact in a two-tier heterogeneous network. The work in [40] (GDSCO) dynamically changes the operating states (on and off) of the small BSs, while keeping the macro BS on with the goal to reduce the network power consumption where users are uniformly distributed in the network. The considered system evaluation is divided into four categories: convergence evaluation, energy efficiency with various network load evaluation, QoS of the macro users associated with the macro BS and offloaded users associated with active small cells, and energy efficiency with dynamic network conditions. Additionally, we consider typical Q-learning and reinforcement learning (RL) proposed in [28] as comparison benchmark for convergence and dynamic network conditions evaluations.

\section{A. Convergence Evaluation}

The convergence of the proposed traffic offloading scheme is evaluated in terms of the traffic load and the achieved energy efficiency. The traffic load is found as a normalized average system loads of all cells during the learning process. This evaluation demonstrates the capability of the proposed scheme 


\begin{tabular}{ll}
\hline Simulation Parameter & Value \\
\hline \hline Bandwidth & $20 \mathrm{MHz}$ \\
Discount factor $(\beta)$ & 0.9 \\
Learning rate $\alpha$ & 0.5 \\
Noise power & $4 \times 10^{-21} \mathrm{~W} / \mathrm{Hz}$ \\
$\epsilon$ & 0.01 \\
Simulation time $d$ & $40 \mathrm{sec}$ \\
Path Loss model & $P L=28.3+22 \log _{10}(d s)$ \\
$p_{c t}$ for macro, pico, femto BS respectively & $130 \mathrm{~W}, 15 \mathrm{~W}, 5 \mathrm{~W}, 0.1 \mathrm{~W}$ \\
Pico BS Tx power & $23 \mathrm{dBm}$ \\
Femto BS Tx power & $14 \mathrm{dBm}$ \\
Macro BS Tx power & $45 \mathrm{dBm}$ \\
\hline
\end{tabular}

Table I

5G ENVIRONMENT SIMULATION PARAMETERS

to maintain the system load of the macrocells at acceptable level as well as the small cells. The average system load during the learning process is potted in Figure 3. The figure shows that our conditional traffic offloading scheme records the minimum average system load compared to the heuristic scheme and the standard. This indicates that the scheme managed to balance the traffic load over the cells in the network. This is due to the conditional offloading that utilizes system load to limit the congestion at the recipient cells. In addition, we plot the achieved average energy efficiency of the network against the number of epoch in Figure 4. The results presented in this figure reveal the advantage of the proposed scheme as it achieved the maximum energy efficiency.

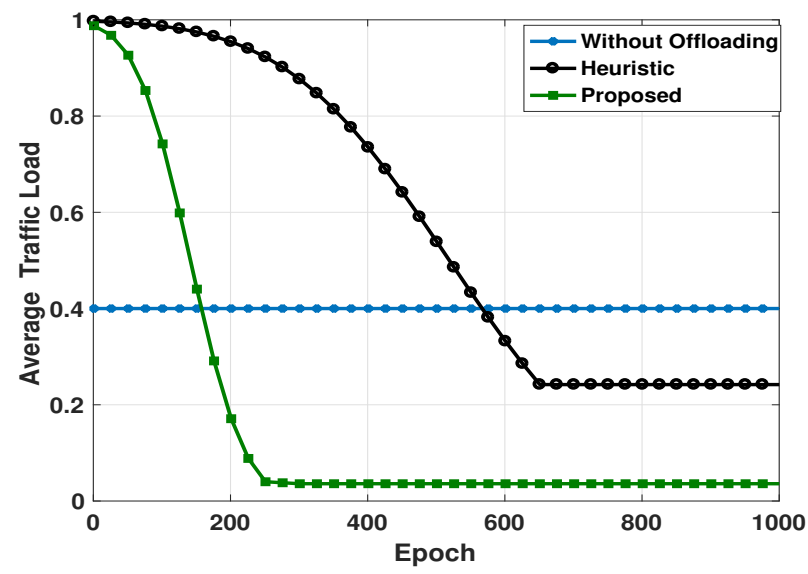

Figure 3. Average system traffic Load

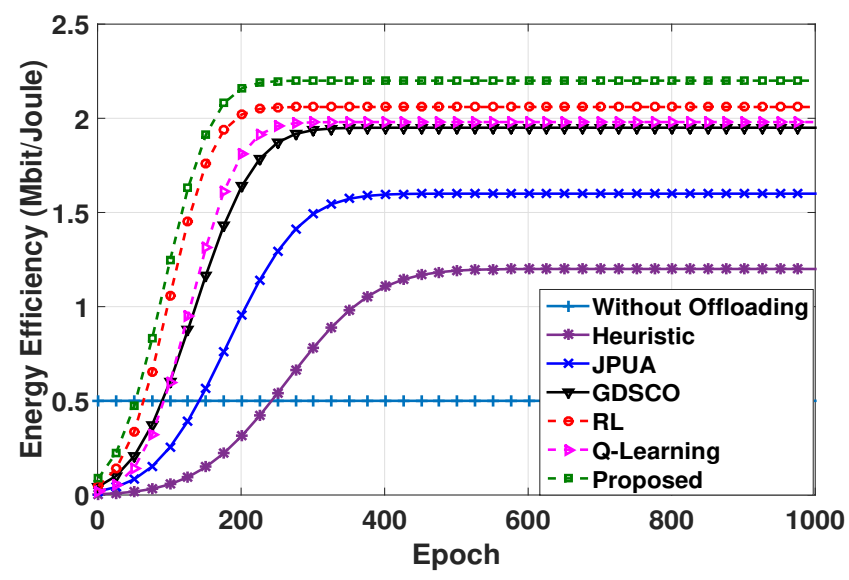

Figure 4. Average system energy efficiency

In addition, we notice that the standard, which does not perform traffic offloading achieves initial low total traffic load as it operates using fewer cells. The average traffic load and energy efficiency achieved by other schemes are not competent to our scheme. The fast convergence of our scheme is due to the impact of the intuitive feature of the proposed online reinforcement learning, which is able to conjecture other macrocells offloading strategies and this reduces the overhead of information exchange among BSs.

\section{B. Energy Efficiency with Various Network Load}

In this part, we evaluate the achieved energy efficiency as a function of the average normalized traffic load of the macrocells and the number of the small cells. The energy efficiency against the normalized traffic load is presented in Figure 5. The figure shows that our scheme recorded the highest energy efficiency compared to other schemes with performance improvement of $14 \%$ over the best competing GDSCO in [40] when the load is high. Moreover, it shows that the energy efficiency decreases as the network load increases. This is due to the fact that with low load, the network experience lower interference, which reduces energy consumption.

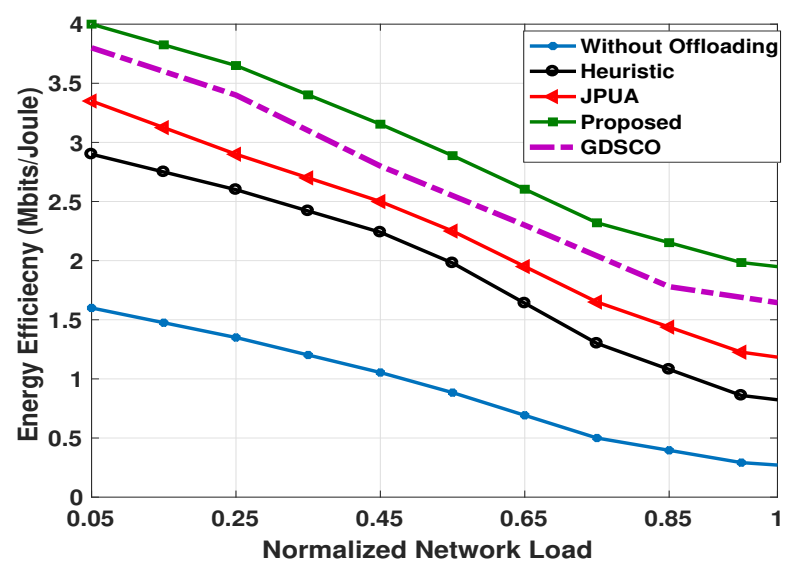

Figure 5. Average system energy efficiency under different network loads

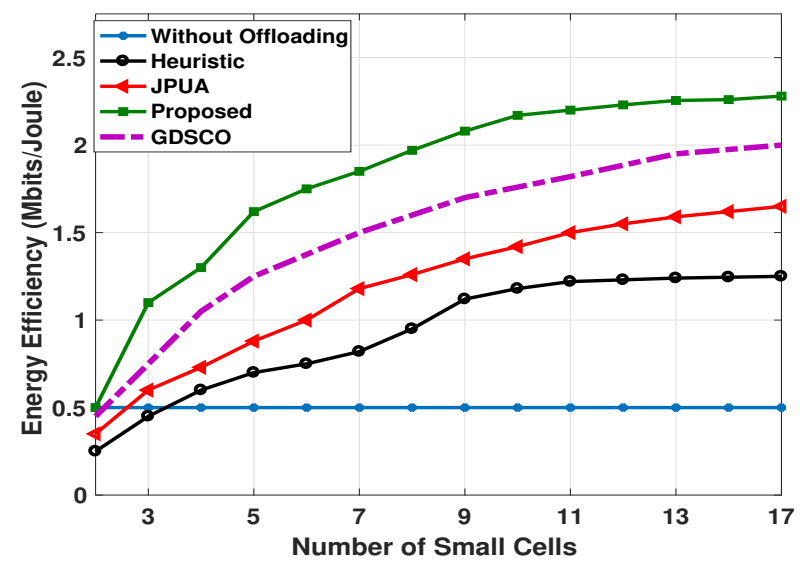

Figure 6. Average system energy efficiency against the number of small cells

Another energy efficiency evaluation is presented in Figure 6 where energy efficiency is plotted against variable number of small cells. The figure demonstrates that the proposed scheme outperforms the other schemes regardless the number of small cells involved. In addition, we can observe that the system 
energy efficiency is improved with the increasing number of small cells. The reason is that small cells enhance the energy efficiency as they consume less power than the macrocells when traffic is offloaded to these cells. However, the energy efficiency saturates at certain number of small cells with little improvement as when the network gets dense, the interference between those cells enforces higher transmission power and consequently more power consumption.

\section{Macro Users QoS Satisfaction}

In this part, we investigate the experience of the macro users as a result of their traffic offloading to small cells. In addition, this evaluation accounts for QoS of the users which are not offloaded and remain associated with the macro BS. The cumulative distribution function (CDF) plots in Figure 7 and Figure 8 present the data rate for macro users associated with the macro BS and the macro users that are offloaded and associate with small cells respectively. For the case when the

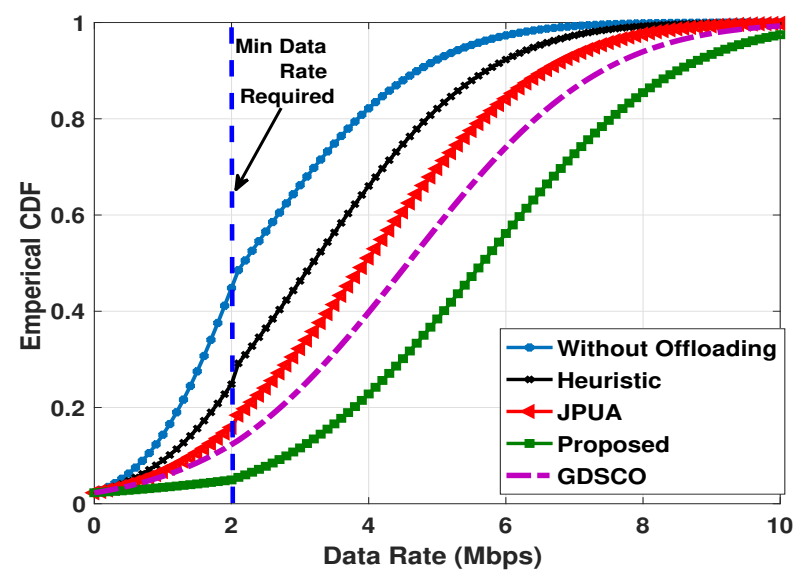

Figure 7. $\mathrm{CDF}$ of data rate for macro users associated with macro BS

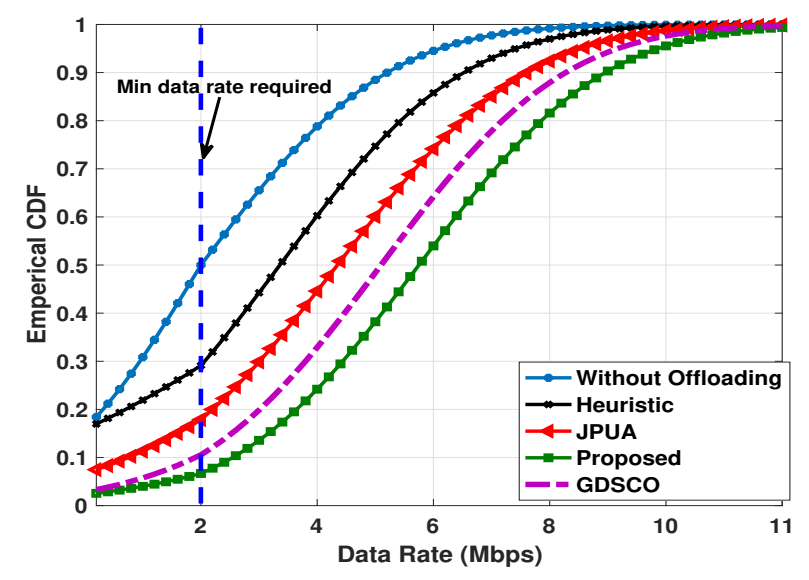

Figure 8. CDF of data rate for macro users offloaded to small BS

network is heavily loaded (i.e. the normalized network load is above 0.8 . The minimum data rate requirements considered in this evaluation is $2 \mathrm{Mbps}$ for the macro user. We notice that the proposed scheme managed to keep the outage (when the user expected data rate is lower than the required rate) at less than $5 \%$ for macro users in both association cases, where other schemes including heuristic based in [38], JPUA in [39], the GDSCO in [40] recorded outage ratio of $25 \%, 16 \%$, and
$12 \%$ respectively for the macro users which remain associated with the macro BS. On the other hand, the outage ratio for the offloaded users for the heuristic, JPUA and GDSCO are $29 \%, 18 \%$, and $11 \%$ respectively as in Figure 8.

The evaluation results in this section indicate that the proposed system load oriented offloading scheme with the intuitive online reinforcement learning improves the offloading process and boosts the performance not only at the level of energy efficiency but also at the level of QoS for the affected users. The proposed intuitive feature enhances the offloading action selection and reduces the network overhead by eliminating the need for information exchange between the network macrocells. Moreover, the evaluation confirms that the system load has a large impact not only on the energy efficiency, but also on users' QoS.

\section{Energy Efficiency with Dynamic Network Conditions}

In this evaluation part, we investigate the impact of dynamic network conditions including various users data rate requirements, variable users density at certain locations, and dynamic channel conditions represented by channel-gain to noise ratio (CNR) on the achieved network energy efficiency. For the diverse macro user data rate demand, we evaluate the performance of the proposed traffic offloading scheme by considering the macro BS located at the upper left corner of Figure 2. This macro BS is located at the center of the cell with four small BSs are located at various coordinates. The group of macro users are randomly located within a plane at which the users are closer to certain small BS than the macro BS. The channel power gains are set according to the specified path loss model, and transmission power of the macro BSs and small BSs as in Table I. Figure 9 presents the achieved energy efficiency as a function of variable macro users data rate requirements. Figure 9 shows the capability of the proposed scheme to maintain the network energy efficiency at the highest level in comparison with other schemes despite the increase in the user data rate demands. We consider typical reinforcement Q-learning without the intuition feature and the reinforcement learning (RL) proposed in [28] in the comparison in addition to other schemes. We notice that the energy efficiency degrades as the users requiremenets increase. This is expected as higher data rate requires higher transmission power of the small BSs and also some of the users might not be offloaded if their requirements exceed the small cell capability. In this case, the overall energy efficiency is degraded.

To evaluate the proposed scheme performance with distinct cells density, we consider an environment with non-uniformly distributed macro users. The macro users density $\chi_{m}$ is evaluated to be within the following range $\left[50 \chi_{0}-\sqrt{3 \varphi^{2}}, 50 \chi_{0}+\right.$ $\left.\sqrt{3 \varphi^{2}}\right]$, where $\varphi$ is the macro user density variation. The range of $\chi_{0}$ is determined according to [41]. Figure 10 depicts the system energy efficiency of the heterogeneous network with variable macrocell density $\left(\chi_{0} / \mathrm{m}^{2}\right)$. The figure shows that our proposed offloading scheme outperforms other schemes including typical reinforcement Q-learning and the RL scheme [28]. The proposed scheme managed to keep the energy 


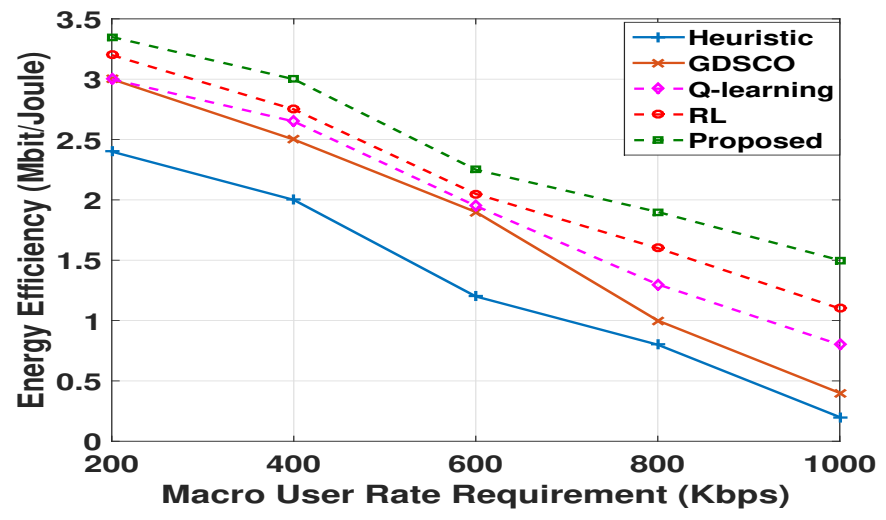

Figure 9. Average system energy efficiency with variable macro user requirement

efficiency at remarkable level despite the efficiency decrease as a result of the raise of the macro users density.

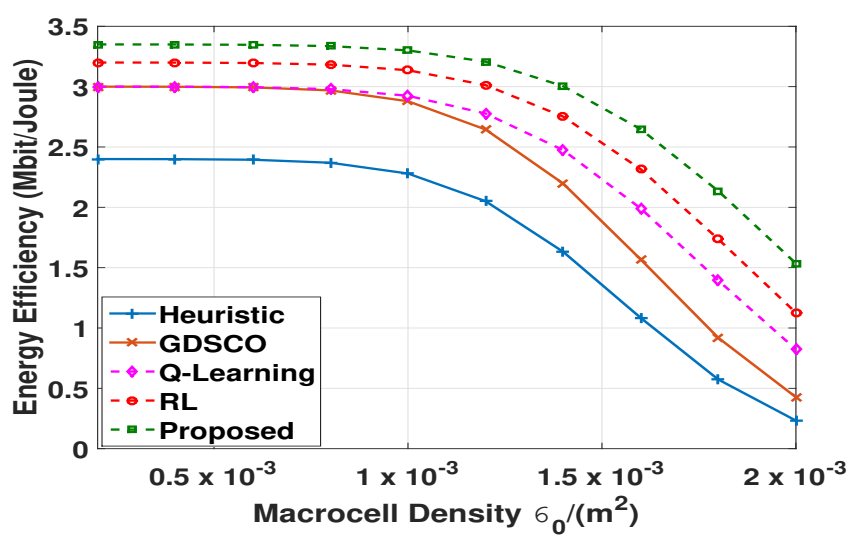

Figure 10. Average system energy efficiency with variable macrocell density $\left(\chi_{0} / m^{2}\right)$

In addition, we have studied the impact of channel conditions alteration on the achieved energy efficiency. Channelgain to noise ratio (CNR) is selected to represent the channel conditions variation. Therefore, we evaluated the impact of CNR variation on the achieved network energy efficiency. The average CNR for macro user $\mathrm{m}$ is defined according to the statistical expectation of the user CNR. We plot the average energy efficiency of the heterogeneous network against the average CNR in Figure 11 with assumption that the remaining simulation parameters are the same. The figure shows that our scheme outperforms all other schemes in terms of the achieved energy efficiency even at low CNR value when poor channel conditions are experienced. We also notice that average energy efficiency is proportional to the CNR.

All the evaluation results confirm the scheme robustness in variable network conditions. The proposed scheme managed to maintain the energy efficiency at a better level than other schemes in spite of the fact that the diverse network conditions severely impact the network performance. Moreover, the results demonstrate the significant benefits from exploiting the developed intuition feature of the online reinforcement learning in traffic offloading decisions.

\section{CONCLUSION}

This paper tackled the traffic offloading problem in multitier $5 \mathrm{G}$ environment with the goal to maximize energy effi-

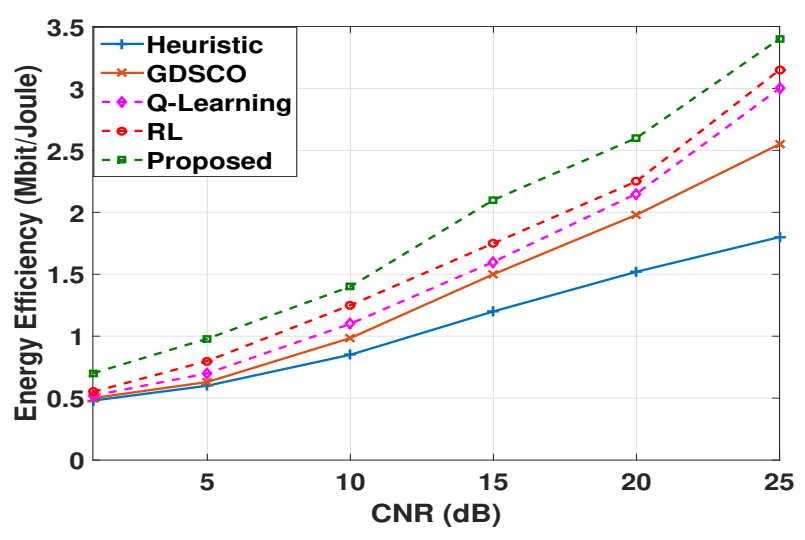

Figure 11. Average system energy efficiency with variable CNR

ciency. The proposed scheme develops a system load oriented traffic offloading scheme using novel intuitive online reinforcement learning methodology to select the traffic offloading strategy that maximizes energy efficiency, guarantees QoS requirements for macro users and maintains acceptable system load. The scheme is applied to heterogeneous network setup with macrocells that perform offloading and picocells and femtocells as recipient cells. The intuitive online reinforcement learning methodology allows the involved macro BSs to conjecture each other offloading strategy without explicit information exchange, which expedites the learning convergence and improves the offloading decision quality. The merits of the proposed scheme are demonstrated in the evaluation results presented in terms of convergence, energy efficiency in dynamic network scenarios, system load maintenance, and users achieved data rate in heavily loaded scenarios.

\section{REFERENCES}

[1] V. Chandrasekhar, J. G. Andrews, and A. Gatherer, "Femtocell networks: a survey”, IEEE Communications Magazine, vol. 46, no. 9, pp. 59-67, September 2008.

[2] L. Hanzo, H. Haas, S. Imre, D. O’Brien, M. Rupp, and L. Gyongyosi, "Wireless myths, realities, and futures: From $3 \mathrm{G} / 4 \mathrm{G}$ to optical and quantum wireless", Proceedings of the IEEE, vol. 100, no. Special Centennial Issue, pp. 1853-1888, May 2012.

[3] G. Biczk, "Economic and ecological impact of ict", 2011.

[4] C. K. Ho, D. Yuan, and S. Sun, "Data offloading in load coupled networks: A utility maximization framework", IEEE Transactions on Wireless Communications, vol. 13, no. 4, pp. 1921-1931, April 2014.

[5] R. Q. Hu and Y. Qian, "An energy efficient and spectrum efficient wireless heterogeneous network framework for 5G systems", IEEE Communications Magazine, vol. 52, no. 5, pp. 94-101, May 2014.

[6] Y. Zhong, X. Ge, H. H. Yang, T. Han, and Q. Li, "Traffic matching in 5G ultra-dense networks", IEEE Communications Magazine, vol. 56, no. 8, pp. 100-105, August 2018.

[7] Y. Zhong, X. Ge, T. Han, Q. Li, and J. Zhang, "Tradeoff between delay and physical layer security in wireless networks", IEEE Journal on Selected Areas in Communications, vol. 36, no. 7, pp. 1635-1647, July 2018.

[8] M. Ajmone Marsan, L. Chiaraviglio, D. Ciullo, and M. Meo, “Optimal energy savings in cellular access networks”, in 2009 IEEE International Conference on Communications Workshops, June 2009, pp. 1-5.

[9] Y. Wu, L. P. Qian, J. Zheng, H. Zhou, and X. S. Shen, "Green-oriented traffic offloading through dual connectivity in future heterogeneous small cell networks", IEEE Communications Magazine, vol. 56, no. 5, pp. 140-147, May 2018.

[10] Waqas ur Rehman, Arshad Hussain, and M. Majid Butt, "Joint user association and BS switching scheme for green heterogeneous cellular network", CoRR, vol. abs/1809.05764, 2018. 
[11] S. Cai, Y. Che, L. Duan, J. Wang, S. Zhou, and R. Zhang, "Green 5G heterogeneous networks through dynamic small-cell operation", IEEE Journal on Selected Areas in Communications, vol. 34, no. 5, pp. 1103 1115, May 2016.

[12] E. H. Hjort Kure, S. Maharjan, S. Gjessing, and Y. Zhang, "A distributed offloading market for $5 \mathrm{~g}$ heterogeneous networks", in 2018 IEEE Global Communications Conference (GLOBECOM), Dec 2018, pp. 1-7.

[13] Y. Wu, X. Yang, L. P. Qian, H. Zhou, X. Shen, and M. K. Awad, "Optimal dual-connectivity traffic offloading in energy-harvesting smallcell networks", IEEE Transactions on Green Communications and Networking, vol. 2, no. 4, pp. 1041-1058, Dec 2018.

[14] S. Zhang, N. Zhang, S. Zhou, J. Gong, Z. Niu, and X. Shen, "Energyaware traffic offloading for green heterogeneous networks", IEEE Journal on Selected Areas in Communications, vol. 34, no. 5, pp. 11161129, May 2016.

[15] W. Cao, G. Feng, S. Qin, and M. Yan, "Cellular offloading in heterogeneous mobile networks with $\mathrm{d} 2 \mathrm{~d}$ communication assistance", IEEE Transactions on Vehicular Technology, vol. 66, no. 5, pp. 42454255, May 2017.

[16] Y. Wu, L. P. Qian, J. Zheng, H. Zhou, and X. S. Shen, "Green-oriented traffic offloading through dual connectivity in future heterogeneous small cell networks", IEEE Communications Magazine, vol. 56, no. 5, pp. 140-147, May 2018.

[17] Rachad Atat, Lingjia Liu, Jinsong Wu, Jonathan Ashdown, and Yang Yi, "Green massive traffic offloading for cyber-physical systems over heterogeneous cellular networks", Mobile Networks and Applications, Feb 2018

[18] B. Zhuang, D. Guo, and M. L. Honig, "Energy-efficient cell activation, user association, and spectrum allocation in heterogeneous networks", IEEE Journal on Selected Areas in Communications, vol. 34, no. 4, pp. 823-831, April 2016

[19] Yue Ling Che, Lingjie Duan, and Rui Zhang, "Dynamic base station operation in large-scale green cellular networks", IEEE J.Sel. A. Commun., vol. 34, no. 12, pp. 3127-3141, Dec. 2016.

[20] A. Bousia, E. Kartsakli, A. Antonopoulos, L. Alonso, and C. Verikoukis, "Multiobjective auction-based switching-off scheme in heterogeneous networks: To bid or not to bid?", IEEE Transactions on Vehicular Technology, vol. 65, no. 11, pp. 9168-9180, Nov 2016.

[21] Jie Wu, Shi Jin, Lei Jiang, and Gang Wang, "Dynamic switching off algorithms for pico base stations in heterogeneous cellular networks", EURASIP Journal on Wireless Communications and Networking, vol 2015, no. 1, pp. 117, 2015

[22] D. Sinha, V. Kavitha, and A. Karandikar, "Load dependent optimal on-off policies in cellular heterogeneous networks", in 2014 12th International Symposium on Modeling and Optimization in Mobile, Ad Hoc, and Wireless Networks (WiOpt), May 2014, pp. 159-166.

[23] Xiaodong Xu, Chunjing Yuan, Jianhui Li, Huixin Zhang, and Xiaofeng Tao, "Reverse auction based green offloading scheme for small cell heterogeneous networks", Mobile Information Systems, vol. 2016, no. 5, pp. 1-10, May 2016

[24] I. AlQerm and B. Shihada, "Enhanced machine learning scheme for energy efficient resource allocation in $5 \mathrm{~g}$ heterogeneous cloud radio access networks", in 2017 IEEE 28th Annual International Symposium on Personal, Indoor, and Mobile Radio Communications (PIMRC), Oct 2017, pp. 1-7.

[25] I. AlQerm and B. Shihada, "Energy-efficient power allocation in multitier 5g networks using enhanced online learning", IEEE Transactions on Vehicular Technology, vol. 66, no. 12, pp. 11086-11097, Dec 2017.

[26] I. AlQerm and B. Shihada, "A cooperative online learning scheme for resource allocation in 5g systems", in 2016 IEEE International Conference on Communications (ICC), May 2016, pp. 1-7.

[27] I. Alqerm and B. Shihada, "Sophisticated online learning scheme for green resource allocation in $5 \mathrm{~g}$ heterogeneous cloud radio access networks", IEEE Transactions on Mobile Computing, vol. 17, no. 10, pp. 2423-2437, Oct 2018

[28] R. Li, Z. Zhao, X. Chen, J. Palicot, and H. Zhang, "Tact: A transfer actor-critic learning framework for energy saving in cellular radio access networks", IEEE Transactions on Wireless Communications, vol. 13, no. 4, pp. 2000-2011, April 2014.

[29] Junhong Ye and Ying Jun (Angela) Zhang, "DRAG: deep reinforcement learning based base station activation in heterogeneous networks", CoRR, vol. abs/1809.02159, 2018.

[30] C. K. Ho, D. Yuan, and S. Sun, "Data offloading in load coupled networks: A utility maximization framework", IEEE Transactions on Wireless Communications, vol. 13, no. 4, pp. 1921-1931, April 2014.

[31] Eunsung Oh, Kyuho Son, and B. Krishnamachari, "Dynamic base station switching-on/off strategies for green cellular networks", IEEE
Transactions on Wireless Communications, vol. 12, no. 5, pp. 2126 2136, May 2013.

[32] L. Gao, G. Iosifidis, J. Huang, and L. Tassiulas, "Economics of mobile data offloading", in 2013 Proceedings IEEE INFOCOM, April 2013, pp. 3303-3308

[33] D. Castanheira, A. Silva, and A. Gameiro, "Limited intersystem information exchange method for heterogeneous networks", IEEE Communications Letters, vol. 19, no. 9, pp. 1656-1659, Sep. 2015.

[34] L. Saker, S. E. Elayoubi, R. Combes, and T. Chahed, "Optimal control of wake up mechanisms of femtocells in heterogeneous networks", IEEE Journal on Selected Areas in Communications, vol. 30, no. 3, pp. 664672, April 2012.

[35] Richard S. Sutton and Andrew G. Barto, Introduction to Reinforcement Learning, MIT Press, Cambridge, MA, USA, 1st edition, 1998.

[36] Christopher J.C.H. Watkins and Peter Dayan, "Q-learning", Machine Learning, vol. 8, no. 3, pp. 279-292, 1992.

[37] Eduardo Rodrigues Gomes and Ryszard Kowalczyk, "Dynamic analysis of multiagent q-learning with $\epsilon$-greedy exploration", in Proceedings of the 26th Annual International Conference on Machine Learning, New York, NY, USA, 2009, ICML '09, pp. 369-376, ACM.

[38] A. Saeed, E. Katranaras, A. Zoha, A. Imran, M. A. Imran, and M. Dianati, "Energy efficient resource allocation for 5G heterogeneous networks", in IEEE 20th International Workshop on Computer Aided Modelling and Design of Communication Links and Networks (CAMAD),, Sept 2015, pp. 119-123.

[39] P. H. Chiang, P. H. Huang, S. S. Sun, W. Liao, and W. T. Chen, "Joint power control and user association for traffic offloading in heterogeneous networks", in IEEE Global Communications Conference, Dec 2014, pp. $4424-4429$.

[40] S. Cai, Y. Che, L. Duan, J. Wang, S. Zhou, and R. Zhang, "Green 5G heterogeneous networks through dynamic small-cell operation", IEEE Journal on Selected Areas in Communications, vol. 34, no. 5, pp. 1103 1115, May 2016.

[41] Y. L. Che, L. Duan, and R. Zhang, "Spatial throughput maximization of wireless powered communication networks", IEEE Journal on Selected Areas in Communications, vol. 33, no. 8, pp. 1534-1548, Aug 2015.

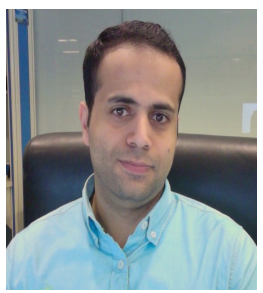

Ismail AlQerm is a Postdoctoral Research Associate and Teaching Professor of computer science at University of Missouri Saint Louis. Ismail acquired his $\mathrm{PhD}$ in computer science from King Abdullah University of Science and Technology (KAUST) in 2018. His research interests include Cognitive radio, Resource allocation in heterogeneous cellular networks, Developing machine learning techniques for resource allocation in wireless networks, and software defined radio prototypes. He is also interested in developing deep learning techniques for resource allocation in big data fields including edge computing and IoT.

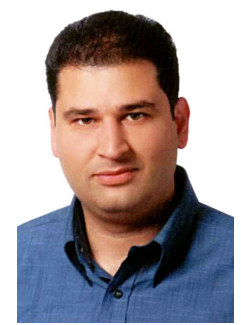

Basem Shihada is an Associate and Founding Professor of computer science and electrical engineering in the Computer, Electrical and Mathematical Sciences and Engineering (CEMSE) Division at King Abdullah University of Science and Technology (KAUST). Before joining KAUST in 2009, he was a visiting faculty at the Computer Science Department in Stanford University. His current research covers a range of topics in energy and resource allocation in wired and wireless communication networks, including wireless mesh, wireless sensor, multimedia, and optical networks. He is also interested in SDNs, IoT, and cloud computing. In 2012, he was elevated to the rank of Senior Member of IEEE. 